\title{
Behavior of Chinchilla lanigera under cage keeping with the introduction of molecular hydrogen into the diet
}

\author{
Elena Panina, Alexey Ivanov, Dmitry Petrov*, Sergey Panteleev \\ Russian State Agrarian University - Moscow Timiryazev Agricultural Academy, Department of \\ Physiology, Ethology and Biochemistry of Animals, 127550 Moscow, Timiryazevskaya street, 49, \\ Russian Federaton
}

\begin{abstract}
Study of the effect of molecular hydrogen on the behavior adaptation of chinchilla in conditions of cage keeping. The experimental animals received drinking water enriched with molecular hydrogen at a dosage of 0.1-1.1 ppm for 10 months. It was found that the peak of activity of the behavior of chinchillas of both groups fell on the period from 19:00 to 9:00. A significant difference between the experimental and control groups were identified by the shape of behavior "movement within the cage" activity in experimental group daytime was higher at 24 minutes $(p<0.05)$ than in the control. The dynamics of daily activity of experimental animals came eh for the period from 19:00 to 08:00 (on average by 12 minutes) and the control group - 22:00-08:00 (an average of 6 minutes). The introduction of drinking water enriched with molecular hydrogen into the diet of the experimental group of chinchillas for 10 months increased the proportion of active behavior of animals by $20.6 \%$.
\end{abstract}

\section{Introduction}

Molecular hydrogen $\left(\mathrm{H}_{2}\right)$ is an antioxidant with favourable physicochemical properties for the body, it is electrically neutral and has a lower molecular weight compared to oxygen. Molecular hydrogen can easily penetrate cell membranes, nuclei, and mitochondria [1]. In addition, molecular hydrogen does not adversely affect physiological processes (temperature, blood pressure, $\mathrm{pH}$ ) [2]. Excess molecular hydrogen is removed from the body during metabolism [3].

Molecular hydrogen effectively neutralizes reactive oxygen species, which negatively affect the body of animals, causing various diseases [4-7]. Foreign scientists in their studies have shown that molecular hydrogen has a positive effect in the treatment of neurological diseases, brain damage, diseases of Alzheimer's, Parkinson's, and others [8, 9]. Studies carried out by K. Nagata confirmed that an abundant intake of water enriched with molecular hydrogen reduced oxidative stress in the brain and prevented the induced stress in mice caused by the physical isolation of animals [10].

\footnotetext{
* Corresponding author: 1941-65@mail.ru
} 
The aim of our work was to study the effect of molecular hydrogen-rich water (HRW) on the behavior of Chinchilla lanigera (long-tailed chinchilla) under cage conditions.

\section{Materials and methods}

For an ethological study, ten pairs of clinically healthy male and female Chinchilla lanigera two months of age with an average weight of 268 grams were selected.

The animals were housed in pairs in separate cages at a constant temperature $\left(19 \pm 2{ }^{\circ} \mathrm{C}\right)$ with 12-hour day/night cycles and free access to food and water. Chinchillas were fed ad libitum with granulated compound feed produced by Ilyinskoye fur farm LLC.

Wooden blocks were placed in each cage for grinding the incisors (gnawing). Watering for both groups of animals was carried out with an automatic drinking system, normalized drinking water with a concentration of mineral impurities in the range of 74-84 ppm with the only difference that an apparatus was connected to the automatic drinking system of the experimental group to generate HRW " Lourdes HS -81". The concentration of molecular hydrogen at the outlet of the nipple drinkers of the experimental group was 0.1-1.1 ppm, depending on the operating mode of the apparatus. The apparatus for generating $\mathrm{H}_{2}$ was turned on twice a day $\left(\mathrm{H}_{2}\right.$ generation mode) in the morning and in the evening for 30 minutes, until the timer turned off, the rest of the time it worked in standby mode.

After 10 months of keeping under the experimental conditions, an ethological study was carried out by the method of remote observation for 24 hours using a video system with four infrared cameras, which made it possible to remotely observe the behavior of animals during the day, including day and night [11-12]. It is important to note that this research method excluded the presence of an observer.

When observing chinchillas, the application "Multitimer" version 2.6.1.217 was used, which had a favorable effect on the quality of the data obtained on the forms of behavior and on their duration [13].

The digital material was processed by the method of variation statistics using the MsExcel program.

\section{Results and discussion}

Studies have shown that the inclusion of water enriched with molecular hydrogen with a hydrogen concentration of 0.1-1.1 ppm in the diet of a Chinchilla lanigera under cage conditions influenced various forms of animal behavior during the day. For inactive forms of behavior (sleep, nap, rest), it was found that in both groups in the first half of the day (daytime) the indicators were higher than in the second half of the day (night time) (Table 1). Also, in the control group, the indicator is higher in the daytime by 43 minutes, and at night by 53 minutes, than in the experimental group. In the daily dynamics, the peak of inactive behavior in both groups fell on 13:00 and was 5-8 minutes in the control group, and 56 minutes in the experimental group (Fig. 1) In general, during the day in the control group, the indicator was higher by 95 minutes, which indicates the fact that the chinchillas of the control group are less active in relation to the experimental group. When analyzing individual forms of inactive behavior, it was revealed that in terms of the form of "rest" behavior in both groups, the indicators are higher in the daytime (Table 1). In the control group, the daily rate is 42.4 minutes higher than in the experimental group. In the diurnal dynamics, the peak in the control group was recorded at 01:00 (36 minutes), and in the experimental group - at 06:00 (38 minutes) (Fig. 2). According to the form of "nap" behavior in both groups, the daily indicator was approximately the same and amounted to 319 minutes. In the diurnal dynamics, the peak in both groups fell at night time (20:00) and 
was 36 minutes in the control group, and 43 minutes in the experimental group. According to the form of "sleep" behavior, the daily indicator in the control group was 42 minutes higher. In the diurnal dynamics, the peak was recorded in the control group at 16:00 and was 42 minutes, and in the experimental group, the peak was at 13:00 (43 minutes).

According to active behavior, which included "food intake", "autocoprophagia", "grooming", "water intake", "moving around the cage", "gnawing a wooden block" in chinchillas of the experimental group during the day, the indicators are higher than in living animals of the control group by 95 minutes $(p<0.05)$ (Table 1$)$. In the diurnal dynamics, the peak of active behavior of chinchillas of both groups occurred at night in the interval from 19:00 to 9:00. The maximum value of active behavior in animals of the experimental group was recorded at 1:00 (duration 38 minutes), and in the control group - at 8:00 (25 minutes) (Fig. 3)

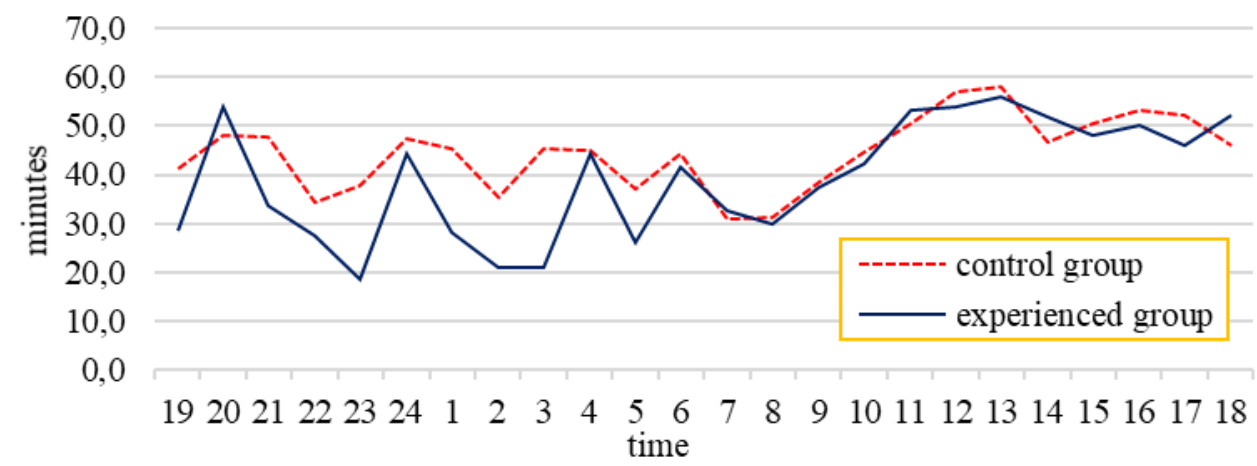

Fig. 1. Daily dynamics of inactive behaviour of Chinchilla lanigera.

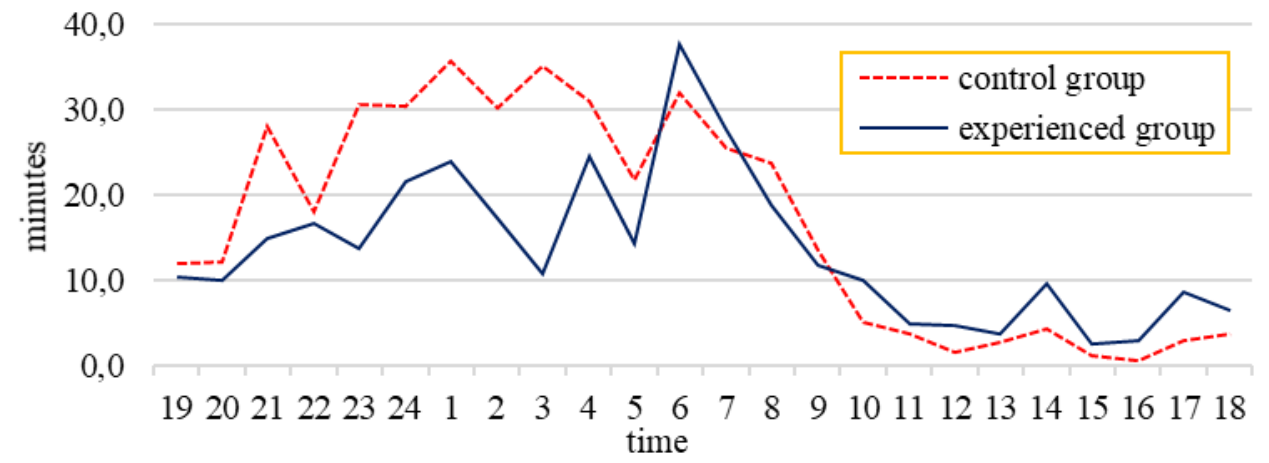

Fig. 2. Daily dynamics of Chinchilla lanigera in the form of rest behaviour.

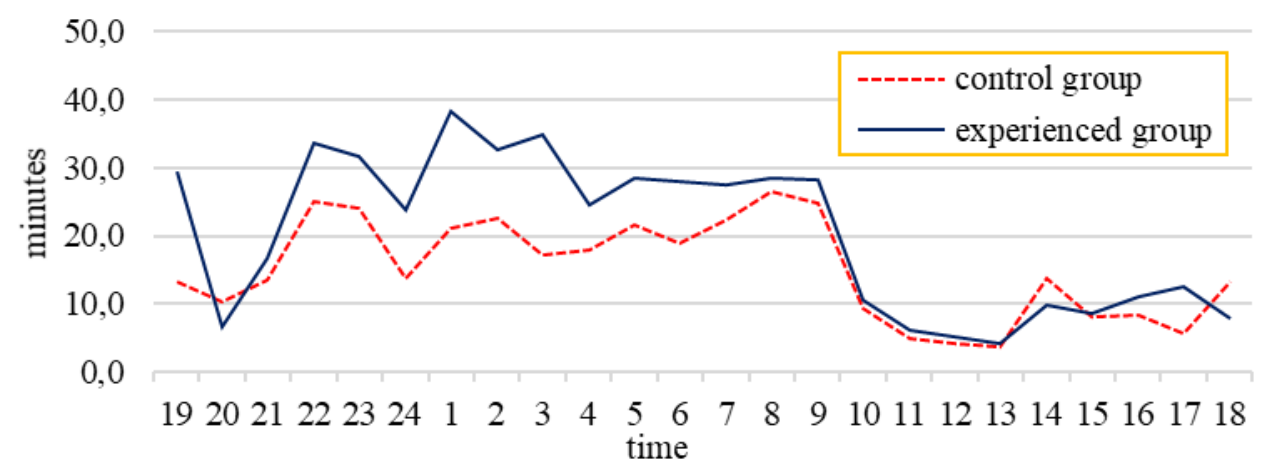

Fig. 3. Daily dynamics of active behaviour of Chinchilla lanigera. 
According to the form of behavior "moving around the cage", the activity of the experimental group in the daytime was higher than in the control one by 24 minutes $(p<0.05)$ (Table 1). In the diurnal dynamics, the period of activity of animals in the experimental group fell on 19: 00-08: 00 (on average 12 minutes), and in the control group - on 22:00-08:00 (on average, 6 minutes). The peak of activity was recorded in the experimental group at 22:00 (19 minutes), and in the control group at 23:00 (12 minutes) (Fig. 4).

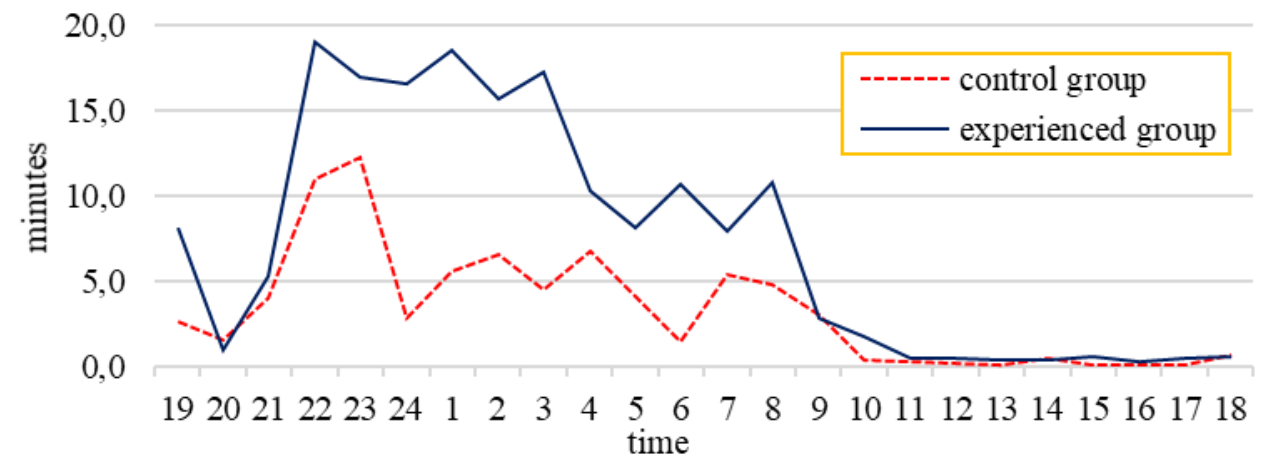

Fig. 4. Daily dynamics of Chinchilla lanigera in the form of movement along the cage behaviour.

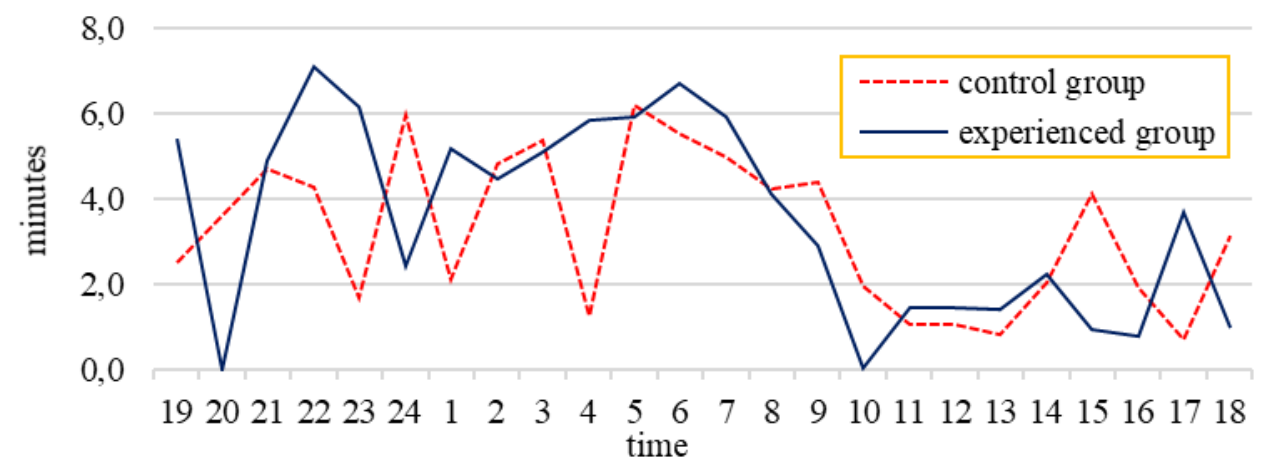

Fig. 5. Daily dynamics of Chinchilla lanigera in the form of feed intake behaviour

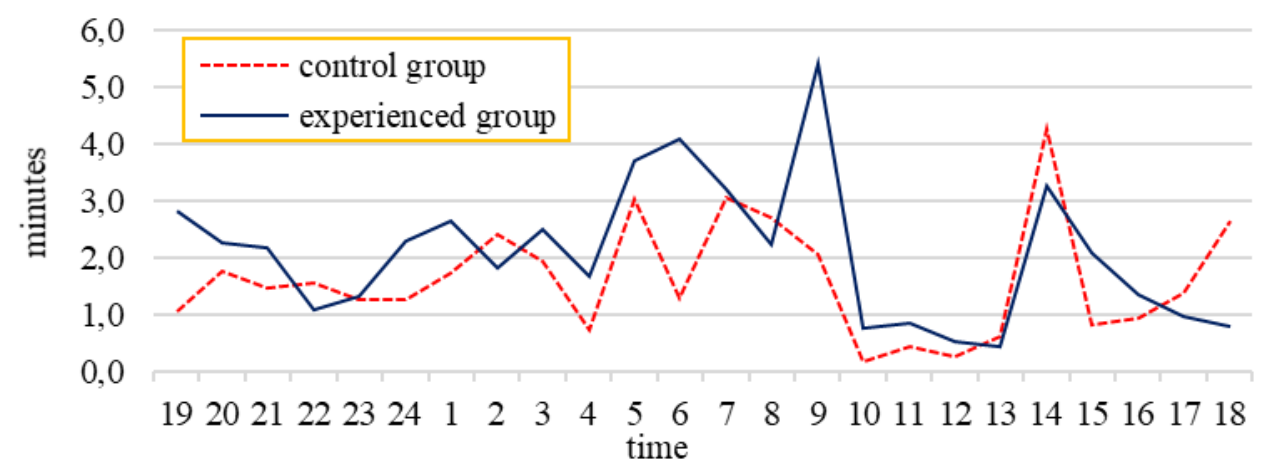

Fig. 6. Daily dynamics of Chinchilla lanigera in the form of grooming

According to their feeding behavior, which included such forms as "food intake", "water intake" and "autocoprophagia", it was found that throughout the entire time the animals periodically showed this type of activity. The maximum value in animals of both groups was recorded at 9:00, but in the experimental group, the feeding behavior was longer and amounted to 16 minutes, which is 3 minutes more than in the control. 
The form of behavior "food intake" was observed at night, while the maximum value in the experimental group was at 22:00 and was 7 minutes, and in the control group at 05:00 and was 6 minutes (Fig. 5). During the day, the animals of the experimental group spent 83 minutes on food intake, and in the control group - 78 minutes, which is 6 minutes more than in the control group (Table 1).

Table 1. Results of monitoring the behaviour of Chinchillas, min.

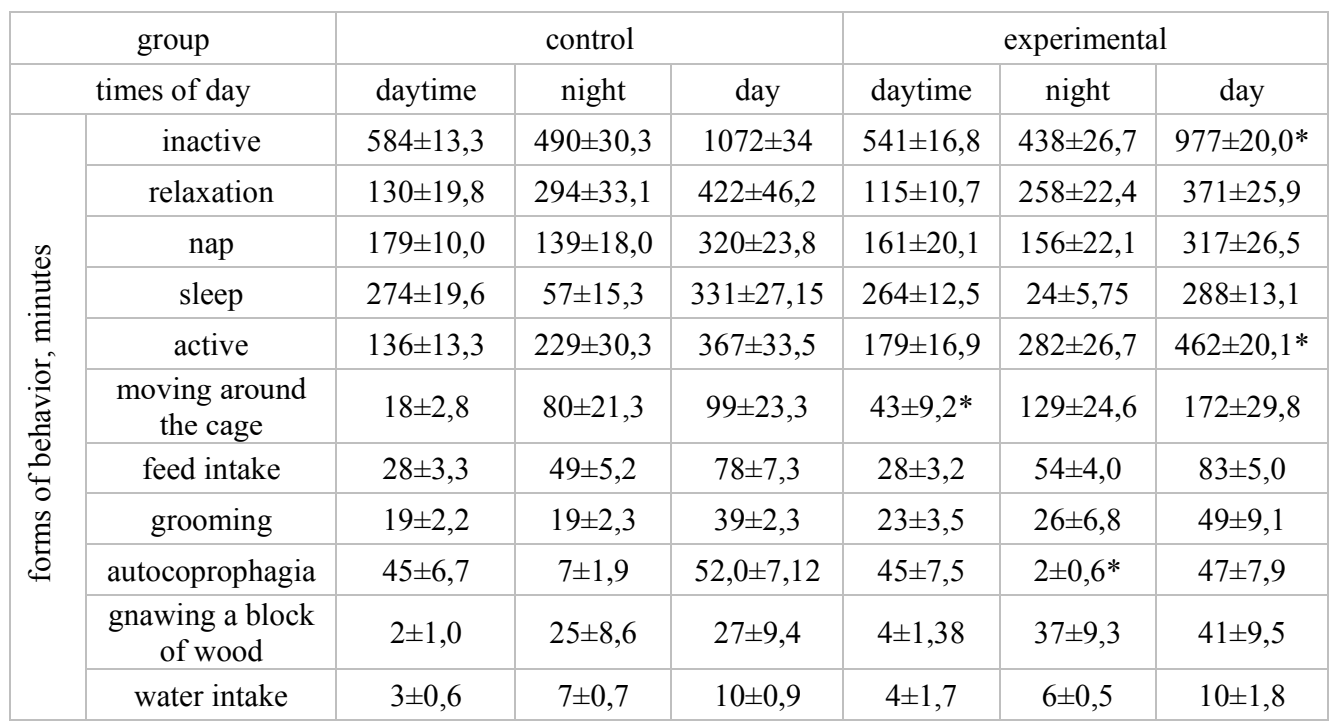

Note: * - the difference between the control and the experimental group with a reliability of $\mathrm{p}<0.05$

According to the form of "water intake" behavior, the activity in the experimental group manifested itself from 21:00-09:00, with a maximum value of 1 minute recorded at 23:00, and in the control group, the maximum value lasted 1 minute 30 seconds, recorded at 06:00 during the period of activity from 20:00-08:00. During the day in both groups, animals spent 10 minutes.

According to the form of behavior "autocoprophagia" it was determined that at night the chinchillas of the control group ate their bolus for 6 minutes, and in the experimental group the indicator was lower for 5 minutes and was 2 minutes $(\mathrm{p}<0.05)$ (Table 1). The peak of this activity of animals fell on the period from 9:00 to 16:00. The maximum value of the experimental and control groups was recorded at 9:00, but for the former it was 13 minutes, and for the latter it was 9 minutes, which is $30.8 \%$ more.

According to the form of behavior, " gnawing a wooden block " during the day, the animals of the experimental group spent 41 minutes, and in the control group - 27 minutes (Table 1). The difference was 14 minutes, which is $34.9 \%$ more. In the diurnal dynamics, the activity of both groups was observed at night from 19:00 to 05:00 in the experimental group, and in the control group - from 23:00 to 06:00. The peak of activity was recorded in the experimental group at 19:00 and was 9 minutes, and in the control group - at 01:00 (8 minutes).

According to the form of grooming behavior in the experimental group, the time of activity during the day was 49 minutes, which is 11 minutes $(21.7 \%)$ more than in the control group. In the diurnal dynamics in the control group, the average activity was 1 minute, and in the experimental group - 2 minutes. The peak of activity in the control group was fixed at 14:00 and amounted to 4 minutes, while in the experimental group the highest rate of activity was at 09:00 (5 minutes) (Fig. 6). 
In percentage terms, the share of inactive behavior in the daily balance of behavior in the control group was $75 \%$, and in the experimental group - $68 \%$, which is $7 \%$ less than in the control group $(\mathrm{p}<0.05)$. Accordingly, in terms of active behavior, the intergroup difference was $7 \%(\mathrm{p}<0.05)$. In the form of behavior "moving around the cage" in the experimental group, the activity is $5 \%$ higher, and in the form of behavior "rest" is lower by $4 \%$ than in the control group (Fig. 7).

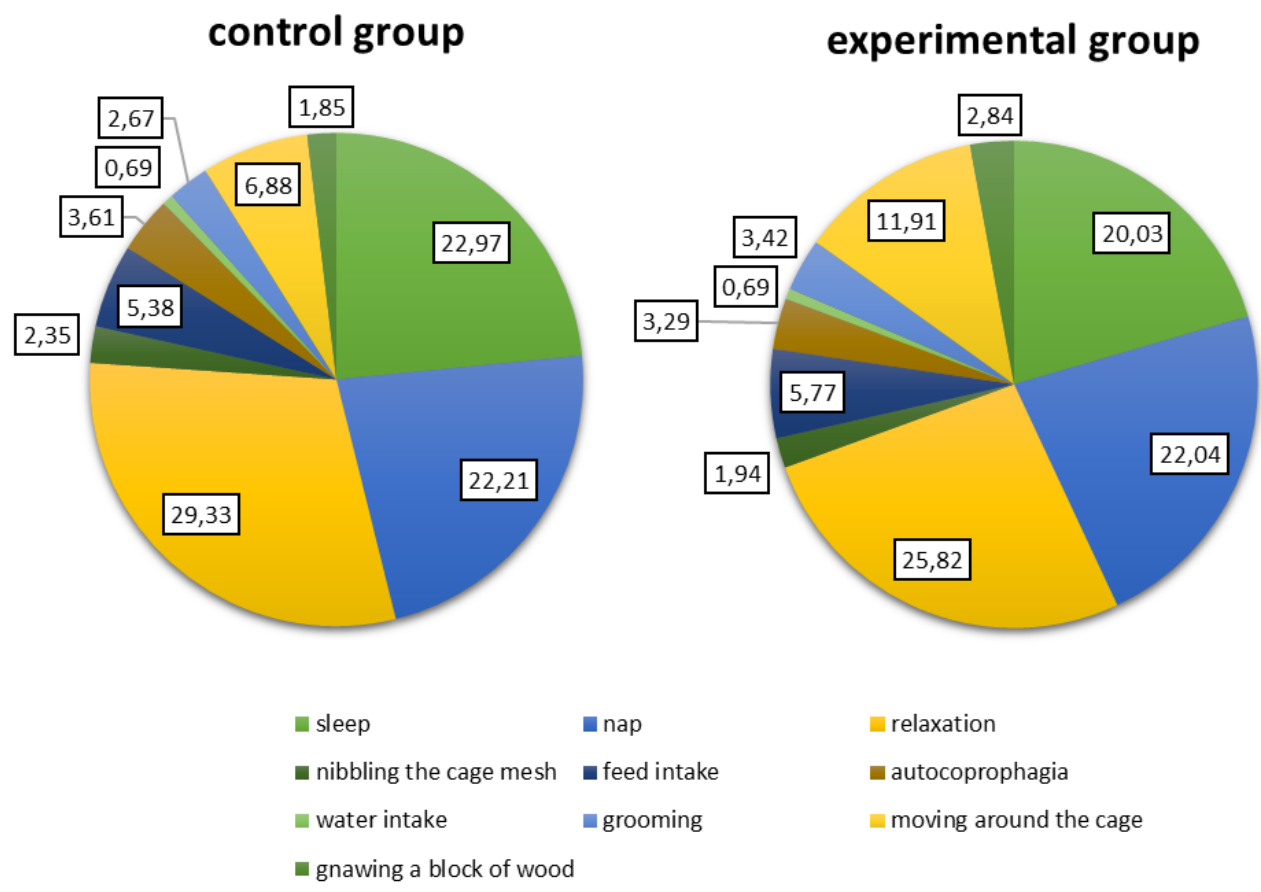

Fig.7. Daily balance of behaviour of Chinchilla lanigera, \%

\section{Conclusion}

Chinchillas of the experimental group who received HRW became $21 \%$ more active than the control group $(p<0.05)$, especially in the form of behavior "moving around the cage", this indicator is $42 \%$ higher than in the control group, especially during the day the time is 24 minutes longer $(p<0.05)$. In the diurnal dynamics of the form of behavior "moving around the cage", the period of activity of animals in the experimental group fell on the period from 19:00 to 08:00 (on average 12 minutes), and in the control group - 22:00-08:00 (on average 6 minutes). According to the form of behavior " gnawing a wooden block " in animals of the experimental group during the day, the activity is $35 \%$ higher. According to the form of grooming behavior, the chinchillas in the experimental group lasted $22 \%$ longer than in the control group. Drinking water, enriched with molecular hydrogen, had a stimulating effect on the active behavior of animals during the day.

\section{References}

1. S. Ohta Biochim. Biophys. Acta Gen. Subj, 1820(5), 586-594 (2012)

2. H. Ono, Y. Nishijima, N. Adachi, M. Sakamoto, Y. Kudo, K. Kaneko, A. Nakao, T. Imaoka. Med. Gas Res, 21 (2012) 
3. A. Tamasawa, K. Mochizuki, N. Hariya, M. Saito, H. Ishida, S. Doguchi, S. Yanagiya, T. Osonoi, Eur. J. Pharmacol, 762, 96-101 (2015)

4. J. Cui. Neuroscience, 335, 232-241 (2016)

5. S. Ohta. Pharmacol. Ther., 144, 1-11 (2014)

6. B. Kura, A.K. Bagchi, P.K. Singal, M. Barancik, T.W. LeBaron, K. Valachova, L. Šoltés, J Slezák, Can. J. Physiol. Pharmacol., 97(4), 287-292 (2019)

7. M. Henry, J. Chambron. Water, 5(4), 2094-115 (2013)

8. A. Shao. Molecular neurobiology, 53, 3462-3476 (2015)

9. C. Wang. Neuroscience letter, 491, 127-132 (2011)

10. K. Nagata, N. Nakashima-Kamimura, T. Mikami, I. Ohsawa, S. Ohta. Neuropsychopharmacology, 34, 501-508 (2009)

11. E.V. Panina, A.A. Ivanov, D.V. Petrov. Rabbit breeding and fur farming, 2, 3-9 (2020)

12. E.V. Panina, A.A. Ivanov, D.V. Petrov. Russian State Agrarian University - Moscow Timiryazev Agricultural Academy, 460-462 (2020)

13. D.V. Petrov, A.A. Ivanov, E.V. Panina. Scientific Life, 15(11), 1551-1562 (2020) 